\title{
A Critical Commentary on the 2017 AGNP Consensus Guidelines for Therapeutic Drug Monitoring in Neuropsychopharmacology
}

\author{
Author \\ Jose de Leon ${ }^{1,2}$ \\ Affiliations \\ 1 Mental Health Research Center at Eastern State Hospital, \\ Lexington, $\mathrm{KY}$, USA \\ 2 Psychiatry and Neurosciences Research Group (CTS-549), \\ Institute of Neurosciences, University of Granada, \\ Granada, Spain, and Biomedical Research Centre in \\ Mental Health Net (CIBERSAM), Santiago Apóstol \\ Hospital, University of the Basque Country, Vitoria, Spain
}

\author{
Key words \\ cytochrome P-450 enzyme system/metabolism, drug \\ interactions, drug monitoring, enzyme induction, enzyme \\ inhibitors, pharmacokinetics, practice guideline, \\ psychopharmacology, psychotropic drugs. \\ $\begin{array}{lr}\text { received } & 17.07 .2017 \\ \text { revised } & 24.07 .2017 \\ \text { accepted } & 31.07 .2017\end{array}$ \\ Bibliography \\ DOI https://doi.org/10.1055/s-0043-117891 \\ Published online: 18.9.2017 \\ Pharmacopsychiatry 2018; 51: 63-68 \\ (c) Georg Thieme Verlag KG Stuttgart · New York \\ ISSN 0176-3679 \\ Correspondence \\ Jose de Leon, M.D. \\ Mental Health Research Center at Eastern State Hospital \\ 1350 Bull Lea Road \\ 40511, Lexington, KY, USA \\ jdeleon@uky.edu
}

\begin{abstract}
In 2004, 2011, and 2017, the Arbeitsgemeinschaft für Neuropsychopharmakologie und Pharmakopsychiatrie (AGNP), a group of German-speaking psychiatric researchers and psychiatrists, published successive versions of therapeutic drug monitoring (TDM) expert group consensus guidelines. The 2017 version has as a major strength its encyclopedic nature, including 1358 references. The guideline has 3 major sections: 1) theoretical aspects of TDM, 2) drug concentration levels in blood to guide neuropsychopharmacotherapy, and 3) practical aspects of TDM in psychiatry and neurology. The writer hopes the time is right for a TDM guideline in psychiatry, which is indicated for: 1) psychiatric researchers ready to value how TDM can contribute to moving psychopharmacology forward, 2) flexible clinicians ready to improve their patient care by personalizing dosing, and 3 ) today's psychiatry residents prepared as a new generation ready to be trained in TDM and willing to continue incorporating TDM as new psychiatric drugs are marketed.
\end{abstract}

\section{Historical Introduction}

\section{From the present to the past and back again}

In 2017, a comprehensive review [1] on antipsychotic blood levels was published in a top US psychiatric journal, reflecting the recent interest of diagnostics companies in entering the market for measuring drug levels; pharmacologists call it therapeutic drug monitoring (TDM). TDM technology for studying multiple psychiatric drugs at the same time (with high throughput analytical methods and automatization) is rapidly advancing and becoming cheaper. Many diagnostics companies are ready to start competing in this potential new market of personalized dosing. Unfortunately, this writer thinks that the science of TDM in psychiatry is developmentally lagging behind the technology used in TDM, and that psychiatrists' training in TDM is definitely underdeveloped. This somewhat pessimistic statement may be surprising from someone who 20 years ago, after using TDM to confirm a drug-drug interaction (DDI) between caffeine and clozapine [2], decided that his clinical and research activities would focus on TDM and pharmacogenetics [3]. The author's 20-year career has been characterized by a struggle with ignorance when dealing with psychiatric journals and thus the need to publish in pharmacological journals. This strug- 
gle was recently validated by a kind, prestigious psychiatric researcher who has never met the author, but who sent a spontaneous e-mail after reading one of his editorials, calling him "almost a voice in the wilderness". This isolation and the need to persevere with his non-mainstream ideas is not risk-free. Only time will tell whether his ideas are wrong, or only in need of the "right" ears of the next generation of psychiatrists willing to listen. Ortega y Gasset, a 20th century Spanish philosopher, in his essay "The Concept of the Generation" argued that a scientific idea need not only be true but also needs to be understood; for that a generational change can be crucial [4].

TDM has a long history in medicine [5]; moreover, psychiatrists and psychiatric researchers were among its pioneers. In 1955, Cade's colleagues, Trautner et al. [6], described lithium TDM as the only way of avoiding lithium toxicity and, in the process, they provided most of the information that contemporary clinicians need concerning lithium TDM in mania. When psychiatrists were trained in the 1980s, lithium and tricyclic antidepressant (TCA) TDM [7] were considered basic tools. Thirty years later, psychiatry residents have very limited familiarity with TDM since lithium and TCAs are underused, and TDM for newer psychiatric drugs is rarely used, except for clozapine, another underused drug. How is it that TDM use has regressed in psychiatry? This writer suspects that it may result from two major overlapping factors: 1) advances in understanding the pharmacokinetic complexity of new psychiatric drugs, and 2) the lack of interest of pharmaceutical companies in dealing with this complexity and their subsequent decision to convince psychiatrists that TDM is not needed for the drugs introduced since the 1990s. The new science of the pharmacokinetics of psychiatric drugs includes complex new concepts such as cytochrome P450 (CYP), inhibitors, inducers, poor metabolizers (PMs) and ultrarapid metabolizers (UMs). The pharmaceutical companies, US academic leaders in psychiatry and many psychiatric journals decided to ignore the advances in these concepts which have been extensively developed in pharmacological journals. The disinterest of US psychiatrists and US psychiatric journals in these concepts is perplexing, since 1) TCAs were crucial in the development of the pharmacokinetic concepts of PM and UM, 2) some of the most important drug metabolism inhibitors are antidepressants, and 3) carbamazepine is one of the most important inducers. Moreover, TDM is the only way to deal with pharmacokinetic variability at a personal level, since the most updated literature can only provide average dosage corrections for an average idealized patient, designed to correct for the average effects of inducers and inhibitors [8].

\section{From the 2004 version to the 2017 version}

In 2004, the Arbeitsgemeinschaft für Neuropsychopharmakologie und Pharmakopsychiatrie (AGNP), a group of German-speaking psychiatric researchers and psychiatrists, published their first TDM expert group consensus guidelines [9] in this journal, Pharmacopsychiatry. In the opinion of this author, the 2004 version did not receive enough attention from US psychiatrists. A bibliographic review verified that most citations of that article have been published by European psychiatric journals [10]. The 2011 update [11] has probably received more attention since, in a ResearchGate search on July 3, 2017, this writer found 407 citations for the 2011 update
(6 years after publication) versus 390 for the 2004 version ( 13 years after publication). This writer is convinced that the 2017 update [12] is being published at an opportune time for becoming highly cited and resurrecting TDM as a major tool for the clinical practice of psychiatry. No other psychiatric organization has tried to publish a similar consensus guideline, although the International League against Epilepsy developed a TDM guideline for antiepileptic drugs (AEDs) in 2008 that has not been updated [13].

\section{Summary}

The 2017 AGNP guideline [12] has 3 major sections: 1) theoretical aspects of TDM, 2) drug concentrations in blood to guide neuropsychopharmacotherapy, and 3) practical aspects of TDM in psychiatry and neurology. It has 7 tables, 7 figures and 1358 references.

\section{Major strength}

Any TDM expert in medicine would need to acknowledge that this third edition of the AGNP is a major accomplishment of encyclopedic magnitude and that no other medical specialty has the luxury of having such a comprehensive consensus guideline. The sophistication of this third version of the AGNP is evident when compared with the 2008 TDM guideline for AEDs [13]. If this writer is correct and TDM is finally ready for resurrection after 30 years, this 2017 guideline is well-timed for helping apply TDM to the treatment of patients by personalizing dosing for psychiatric drugs, including not only first-generation psychiatric drugs (which started in the 1980s) but also second-generation psychiatric drugs.

\section{Weaknesses}

This section discusses weaknesses at the methodological level in the development of pharmacokinetic guidelines in general, and then focuses on weaknesses of the AGNP guideline at the levels of methodology, content and dissemination.

\section{Weaknesses in methodology in the development of pharmacokinetic guidelines in general}

In the view of this writer [14], there are two major types of scientific thinking in medicine: mathematical thinking, which is currently mainly expressed by the evidence-based medicine (EBM) approach, and mechanistic thinking, which is mainly followed by basic scientists, such as pharmacologists or physiologists. Medical mathematical thinkers, such as loannidis [15], want experts to develop guidelines following principles of EBM such as those applied by the Cochrane library. Unfortunately, these principles assume that patients can be represented by the average patient, while methods for personalizing dosing, such as TDM, are most effective with patients who are not average, whom statisticians call outliers [16]. This writer acknowledges that any pharmacokinetic guideline (including the AGNP guideline) is necessarily heavily dependent on pharmacokinetic mechanisms, and as such may look methodologically weak to medical scientists firmly committed to EBM principles. On the other hand, EBM experts may not grasp 1) the peculiarity of pharmacokinetic mechanisms and 2) the limitations of EBM thinking when used in TDM studies. 
Lack of understanding pharmacokinetic mechanisms

EBM scientists are not familiar with pharmacokinetic mechanisms and with the inherent limitations of TDM literature, which is not supported by commercial companies with deep pockets paying for double-blind randomized clinical trials (RCTs) as EBM dictates. Pharmacokinetic mechanisms are certainly very peculiar, since evolution can produce remarkably peculiar things, but pharmacokinetic mechanisms do follow laws; they are not statistical laws, but pharmacokinetic laws, such the therapeutic window law [17] which can be used to predict atomoxetine TDM results and suggests dosing changes beyond those approved by the FDA after the publication of the RCTs by the commercial company $[18,19]$.

Lack of understanding the limitations of traditional statistical thinking in TDM

A recent article on clozapine dosing published using the Cochrane methodology [20] is excellent proof of how using EBM methodology can provide results that make no sense in the context of pharmacokinetics. Indeed, if you know pharmacokinetics, you know that the question of which clozapine dose is best is essentially nonsensical. There is no good "average" clozapine dose; the appropriate clozapine dose: 1 ) is definitely dependent on a) smoking status and $b$ ) the presence or absence of co-prescriptions of inducers or inhibitors, and 2) possibly dependent on a) gender, b) East Asian ancestry, c) CYP2C19 PM status, and d) presence or absence of inflammation [21]. As none of the clozapine RCTs were stratified by any of these confounding factors, no published article includes information on clozapine dosing after accounting for these confounders. The authors of this EBM clozapine review concluded, "It is unclear, however, what dose of clozapine is most effective with the least side effects." [20]. In reality, there is no need to make the effort to complete such a review to reach that conclusion; a pharmacokinetic scientist without completing any EBM review can explain that the only way to establish the clozapine dosage for a specific patient is to complete TDM and then reference the AGNP clozapine therapeutic reference range. Unfortunately, these experts on Cochrane methodology [20] are much less sophisticated in their knowledge of TDM and do not know that the 2004 [9] or 2011 AGNP guidelines [11] exist; they preferred to quote a 1995 study on clozapine's therapeutic reference range.

\section{Weaknesses in the development of the AGNP guide- line}

As advanced in the prior section, an EBM scientist may not be impressed by Section 2.7 of the 2017 AGNP guideline definitions on the levels of utility used to classify the TDM findings. He/she would question the nearly complete absence of double-blind RCTs and meta-analyses used in the development of this guideline. Despite the lack of regard for EBM principles that this author shares with the AGNP, he cannot deny that the lack of careful discussion of whether or not to apply classic EBM principles to these AGNP TDM guidelines appears to be a legitimate critique. Clearly, this writer, the AGNP and other TDM experts need to develop innovative ways of improving the methodology of TDM systematic reviews and meta-analyses [22] by developing a new system of quality scores based on pharmacokinetic principles instead of typical EBM principles such as blinding, since TDM studies by nature have imbibed some level of blinding [22].

\section{Weaknesses in the AGNP guideline's content}

This writer can find multiple small details with which to disagree with the AGNP authors regarding Table 1 (involved enzymes), Table 2 (inhibitors) and Table 3 (inducers), but these disagreements may be more fairly classified as weaknesses in the science of pharmacokinetics rather than weaknesses in the AGNP guideline. Pharmacokinetic science is severely underdeveloped, particularly for drugs marketed before 1996 [23]. Published articles from various authors provide conflictive information on psychiatric drugs regarding metabolic enzymes, inhibitors and inducers, including the possibility of false positive findings promoted by pharmaceutical companies [24, 25].

\section{Weaknesses in the AGNP guideline's dissemination}

An objective critic may claim that if past AGNP guidelines are as wonderful as this writer proposes, why have they had limited impact in the international psychiatric literature and been ignored by US psychiatry, the world leader? As previously described, the same critique applies to the 20 years of research on pharmacogenetics and TDM by this writer [3]. The truth is that pharmacogenetics was mainly developed in continental Europe [26] and the AGNP guidelines $[9,11,13]$ were developed by German-speaking psychiatric scientists. US psychiatry tends to ignore findings described by nonUS psychiatrists, particularly from non-English speaking countries. For the last 20 years, US psychiatric textbooks and scientific authors have focused on pharmacodynamic mechanisms, although we have very limited, if any, understanding of them to the point that pharmaceutical companies have recently decided that pharmacodynamic mechanisms in psychiatry are too complex [27] and thus it is better for them to abandon psychiatric drug development (the reader may comment ironically, "after making a ton of money"). On the other hand, psychiatric textbooks and journals have ignored the pharmacokinetic science described in pharmacological journals. The outcome of two recent reviews on AED DDIs [28, 29] confirms this point about the status of pharmacokinetic science versus pharmacodynamic science in psychiatry. The first review focused on pharmacokinetic DDIs [28] and described dose correction factors to orient clinicians; these presented no great problems to the journal reviewers since they were based on some TDM and other pharmacokinetic studies, while the second and analogous review of pharmacodynamic DDIs [29] was unmercifully criticized by the reviewers. These critics were not unjustified since the described pharmacodynamic DDIs were based on almost no published data, but only on clinical experience and speculations based on the little that we know of pharmacodynamic mechanisms [29]. This writer had to remind the reviewers that lack of publication does not protect patients from pharmacodynamic DDIs since patients can experience DDIs even if they have never been published. To conclude, this writer would like to think that the lack of impact of the AGNP guideline and pharmacokinetic science in US psychiatry is not because he and the AGNP authors are completely incompetent in marketing, but because they were working against the powerful efforts of pharmaceutical companies and US academic psychiatrists who 
promoted simplistic pharmacodynamic speculations while trying to negate the much more reliable science of pharmacokinetics and the value of TDM.

\section{Possible Additional Articles to follow this Guideline in the Immediate Future}

This writer believes that the herculean task performed by Hiemke et al. [12] deserves to be used extensively to help develop other articles. Initially and most importantly, Hiemke et al. [12] need to select, among the extensive list of authors, some who are both MDs and practicing clinicians, and who would be willing to write a simplified version for extremely busy clinicians which 1) is as short as possible, 2) focuses only on practical aspects, and 3) eliminates any mathematical formula which tends to be aversive for clinicians. Other subproducts may be generated by using the principles of pharmacokinetic science. In that sense, it would be interesting to use the AGNP therapeutic reference range to explore which psychiatric drugs have wide and which have narrow therapeutic ranges or indexes [17].

\section{Future Developments}

This 2017 update is being published in the midst of major changes in the practice of medicine and psychiatry, which increases the difficulty in predicting how TDM developments will be influenced by advances in technology, pharmacokinetic science, computerized auxiliary tools and medical education.

\section{Technology}

The 2017 AGNP guideline [12] briefly mentions (in Section 3.4) the advances in laboratory assays and using samples other than blood. This writer is aware of several companies using proprietary new TDM technology about which no article has been published but commercialization is being explored. He has no clue whether or not these companies or technologies will revolutionize psychiatric TDM in the next five years.

\section{Pharmacokinetic science}

This writer has the fantasy that the unbiased and open-minded review of the unusual TDM results of some of his most unusual patients could lead to advances in pharmacokinetic science that may help advance personalized dosing in psychiatry. In the last few years, he has proposed that: 1 ) valproate may be an inducer [30] and an auto-inducer [30,31]; 2) some new AEDs may be mild inducers, but at times clinically relevant inducers [30], including oxcarbazepine which in high doses ( $\geq 1200 \mathrm{mg} /$ day) can induce other drugs causing adverse drug reactions (ADRs) after discontinuation [32] or lack of efficacy after addition [33]; 3) some patients may be particularly sensitive to carbamazepine, phenytoin or phenobarbital requiring massive doses of some medications (e. g.; $1600 \mathrm{mg} /$ day of lamotrigine) [34, 35]; and 4) protein binding and non-linear kinetics are crucial in understanding valproate TDM [31,36]. These findings have not yet been replicated by other authors, and are looked upon with suspicion by traditional pharmacologists. Only time and replication will tell whether these are innovative findings that must be included in the next AGNP update, or are merely the seemingly delusional ideas of this writer. It cannot be ruled out that this writer has spent too much time "obsessing" about justifying with pharmacokinetic concepts his "crimes" from 20 years ago when he was clueless about valproate pharmacokinetics and decided to prescribe $10,500 \mathrm{mg} /$ day of valproate to obtain therapeutic concentrations in one of his patients [31].

\section{Computerized auxiliary tools}

Some of the younger authors of the AGNP guideline should contemplate how this guideline could be transformed into software tools to facilitate its use by younger clinicians. This writer tends to think that any computerized approach that makes TDM more friendly in the real world needs to maintain the complexity of pharmacological response in the real world, and account for the fact that TDM can be influenced by genetic, environmental and personal variables [37].

\section{Medical education}

Finally, the most important pending issue in TDM is how to educate psychiatrists and other clinicians so that they can take advantage of these updated guidelines [12]. The AGNP guideline Section 3.5. 1 provides 3 patient cases with TDM but these boxes are only a small educational effort. After many years of attempting it, this writer is not optimistic about teaching TDM to practicing clinicians, so he developed a PowerPoint course for teaching residents about pharmacodynamic and pharmacokinetic thinking using multiple examples of TDM from real patients [38].

\section{Conclusion}

To conclude, this writer hopes the time is right for TDM guidelines in psychiatry, which are indicated for: 1) psychiatric researchers ready to value how TDM can contribute to moving psychopharmacology forward, 2) flexible clinicians ready to improve their patient care by personalizing dosing, and 3) today's psychiatry residents prepared as a new generation ready to be trained in TDM just as those trained in the 1980s, but this new generation may be willing to continue to incorporate TDM as new psychiatric drugs are marketed. It has been proposed that just as a global positioning system (GPS) is crucial for modern navigation, TDM may be crucial for modern clinical pharmacology [39].

\section{Acknowledgments}

Lorraine Maw, M.A., from the Mental Health Research Center, Eastern State Hospital, Lexington, KY, USA, helped edit this article.

\section{Conflict of interest and sources of funding}

No commercial organizations had any role in the writing of this paper for publication. Dr. de Leon personally develops his presentations for lecturing, has never lectured using any pharmaceutical or pharmacogenetic company presentation, and has never been a consultant for pharmacogenetic or pharmaceutical companies. In the past, Dr. de Leon has received researcher-initiated grants from Eli Lilly (one ended in 2003 and the other, as co-investigator, ended in 2007); from Roche Molecular Systems, Inc. (ended in 2007); and, in collaboration with Genomas, Inc., 
from the NIH Small Business Innovation Research program (ended in 2010). He has been on the advisory boards of Bristol-Myers Squibb (2003/04) and AstraZeneca (2003). Roche Molecular Systems supported one of his educational presentations, which was published in a peerreviewed journal (2005). His lectures have been supported once by Sandoz (1997, at that time the marketer of clozapine), twice by Lundbeck (both in 1999), twice by Pfizer (both in 2001), three times by Eli Lilly (once in 2003 and twice in 2006), twice by Janssen (2000 and 2006), once by Bristol-Myers Squibb (2006), and seven times by Roche Molecular Systems, Inc. (once in 2005 and six times in 2006). Dr. de Leon may have some conflict of interest due to personal relationships with some of the authors of the AGNP guidelines. After the publication of the first version of the AGNP consensus guideline, Dr. de Leon contacted Dr. Hiemke by e-mail. Since 2004, Dr. de Leon has continued, through e-mail and phone, a friendly relationship with Dr. Hiemke although they have never met in person. As they have collaborated in three published articles and share interest in therapeutic drug monitoring, it would be naïve for Dr. de Leon to state that his judgment on any of Dr. Hiemke's articles can be completely free of bias. Dr. de Leon has also developed a friendly relationship by e-mail and/or other personal contacts with Drs. Stingl and Baumann and has collaborated with Dr. Schoretsanitis in one article. Dr. de Leon is not aware of any other possible conflict of interest with the rest of the authors of the AGNP consensus guideline.

\section{References}

[1] Horvitz-Lennon M, Mattke S, Predmore Z et al. The role of antipsychotic plasma levels in the treatment of schizophrenia. Am J Psychiatry 2017; 174: 421-426

[2] Odom-White A, de Leon J. Clozapine levels and caffeine. J Clin Psychiatry 1996; 57: 175-176

[3] de Leon J. Pharmacogenetic tests in psychiatry: From fear to failure to hype. J Clin Psychopharmacol 2016; 36: 299-304

[4] Ortega y Gasset J. The concept of the generation. In the modern theme. New York: Harper \& Row; 1961: 11-18

[5] Pippenger CE. Therapeutic drug monitoring assay development to improve efficacy and safety. Epilepsy Res 2006; 68: 60-63

[6] Trautner EM, Morris R, Noack CH et al. The excretion and retention of ingested lithium and its effect on the ionic balance of man. Med J Aust 1955; 42: 280-291

[7] No authors listed Tricyclic antidepressants-blood level measurements and clinical outcome: An APA Task Force report. Task force on the use of laboratory tests in psychiatry. Am J Psychiatry. 1985; 142: 155-162

[8] Spina E, Hiemke C, de Leon J. Assessing drug-drug interactions through therapeutic drug monitoring when administering oral secondgeneration antipsychotics. Expert Opin Drug Metab Toxicol 2016; 12: 407-422

[9] Baumann P, Hiemke C, Ulrich S et al. Arbeitsge-meinschaft fur neuropsychopharmakologie und pharmakopsychiatrie. The AGNP-TDM expert group consensus guidelines: Therapeutic drug monitoring in psychiatry. Pharmacopsychiatry 2004; 37: 243-265

[10] Schäfer A, Hiemke C, Baumann P. Consensus guideline for therapeutic drug monitoring in psychiatry (2004): Bibliometric analysis of citations for the period 2004-2011. Nord J Psychiatry 2016; 70: 202-207

[11] Hiemke C, Baumann P, Bergemann N et al. AGNP consensus guidelines for therapeutic drug monitoring in psychiatry: Update 2011. Pharmacopsychiatry 2011; 44: 195-235

[12] Hiemke C, Bergemann N, Clement HW et al. Consensus guidelines for therapeutic drug monitoring in neuopsychopharmacology: update 2017. Pharmacopsychiatry 2018; 51: 9-62
[13] Patsalos PN, Berry D], Bourgeois BF et al. Antiepileptic drugs-best practice guidelines for therapeutic drug monitoring: a position paper by the subcommission on therapeutic drug monitoring, ILAE Commission on Therapeutic Strategies. Epilepsia 2008; 49: 1239-1276

[14] de Leon J, De Las Cuevas C. The art of pharmacotherapy: Reflections on pharmacophobia. J Clin Psychopharmacol 2017; 37: 131-137

[15] Ioannidis JPA. Hijacked evidence-based medicine: Stay the course and throw the pirates overboard. J Clin Epidemiol 2017; 84: 11-13

[16] de Leon J. Evidence-based medicine versus personalized medicine: Are they enemies? J Clin Psychopharmacol 2012; 32: 153-164

[17] de Leon J. The crucial role of the therapeutic window in understanding the clinical relevance of the poor versus the ultrarapid metabolizer phenotypes in subjects taking drugs metabolized by CYP2D6 or CYP2C19. J Clin Psychopharmacol 2007; 27: 241-245

[18] de Leon J. Translating pharmacogenetics to clinical practice: Do cytochrome P450 2D6 ultrarapid metabolizers need higher atomoxetine doses? J Am Acad Child Adolesc Psychiatry 2015; 54: 532-544

[19] Brown JT, Abdel-Rahman SM, van Haandel L et al. Single dose, CYP2D6 genotype-stratified pharmacokinetic study of atomoxetine in children with ADHD. Clin Pharmacol Ther 2016; 99: 642-650

[20] Subramanian S, Völlm BA, Huband N. Clozapine dose for schizophrenia. Cochrane Database Syst Rev 2017; 6 CD009555

[21] Spina E, de Leon J. Clinical applications of CYP genotyping in psychiatry. J Neural Transm (Vienna) 2015; 122: 5-28

[22] Schoretsanitis G, Spina E, Hiemke $C$ et al. A systematic review and combined analysis of therapeutic drug monitoring studies for long-acting risperidone. Expert Review in Clinical Pharmacology 2017, doi:10.1080/17512433.2017.1345623

[23] de Leon J. Highlights of drug package inserts and the website DailyMed: the need for further improvement in package inserts to help busy prescribers. J Clin Psychopharmacol 2011; 31: 263-265

[24] de Leon J. False-negative studies may systematically contaminate the literature on the effects of inducers in neuropsychopharmacology. Part I: focus on epilepsy. J Clin Psychopharmacol 2014; 34: 177-183

[25] de Leon J. False-negative studies may systematically contaminate the literature on the effects of inducers in neuropsychopharmacology: Part II: Focus on bipolar disorder. J Clin Psychopharmacol 2014; 34: 291-296

[26] Meyer UA. Pharmacogenetics - five decades of therapeutic lessons from genetic diversity. Nat Rev Genet 2004; 5: 669-676

[27] Fibiger HC. Psychiatry, the pharmaceutical industry, and the road to better therapeutics. Schizophr Bull 2012; 38: 649-650

[28] Spina E, Pisani F, de Leon J. Clinically significant pharmacokinetic drug interactions of antiepileptic drugs with new antidepressants and new antipsychotics. Pharmacol Res 2016; 106: 72-86

[29] Spina E, de Leon J. Potentially clinically relevant pharmacodynamics drug interactions between antiepileptic and psychotropic medications: An update. Curr Pharm Des 2018 in press

[30] de Leon J. The effects of antiepileptic inducers in neuropsychopharmacology, a neglected issue. Part I: A summary of the current state for clinicians. Rev Psiquiatr Salud Ment 2015; 8: 97-115

[31] Jackson J, McCollum B, Ognibene J et al. Three patients needing high doses of valproic acid to get therapeutic concentrations. Case Rep Psychiatry 2015; 2015: 542862

[32] O’Neill A, de Leon J. Two case reports of oral ulcers with lamotrigine several weeks after oxcarbazepine withdrawal. Bipolar Disord 2007; 9: 310-313

[33] McGrane IR, Loveland JG, de Leon J. Possible oxcarbazepine inductive effects on aripiprazole metabolism: a case report. J Pharm Pract 2017; 1: 897190017710523 [Epub] 
[34] de Leon J. The effects of antiepileptic inducers in neuropsychopharmacology, a neglected issue. Part II: Pharmacological issues and further understanding. Rev Psiquiatr Salud Ment 2015; 8: 167-188

[35] Jackson J, McCollum B, O'Neill A et al. A case report that suggested that the patient was very sensitive to induction and needed 1600 $\mathrm{mg} / \mathrm{d}$ of lamotrigine to get therapeutic concentrations. J Clin Psychopharmacol 2015; 35: 745-747

[36] Lana F, Martí-Bonany J, Fuster J et al. Reduction in serum concentration of valproic acid secondary to the intake of ibuprofen as an example of valproic acid auto-induction metabolism. Actas Esp Psiquiatr 2016; 44: 136-144
[37] Crettol S, de Leon J, Hiemke C et al. Pharmacogenomics in psychiatry: From therapeutic drug monitoring to genomic medicine. Clin Pharmacol Ther 2014; 95: 254-257

[38] de Leon J. Training psychiatrists to think like pharmacologists. http:// inhn.org/home/courses/jose-de-leon-training-psychiatrists-to-thinklike-pharmacologists.html

[39] Buclin T, Gotta V, Fuchs A et al. Monitoring drug therapy. Br J Clin Pharmacol 2012; 73: 917-923 